\title{
Alterations of Brain Activity Associated With Resolution of Emotional Distress and Pain in a Case of Severe Irritable Bowel Syndrome
}

\author{
DOUGLAS A. DROSSMAN,* YEHUDA RINGEL,* BRENT A. VOGT, ${ }^{\ddagger}$ JANE LESERMAN,* WEILI LIN, $\$$ \\ J. KEITH SMITH,§ and WILLIAM WHITEHEAD* \\ UNC Center for Functional GI and Motility Disorders, *Division of Digestive Diseases and \$Department of Radiology and Biomedical \\ Engineering, University of North Carolina, Chapel Hill, North Carolina; and ${ }^{\ddagger}$ Cingulum NeuroSciences Institute and Department of \\ Neuroscience and Physiology, SUNY Upstate Medical Center, Syracuse, New York
}

Background \& Aims: The association of psychosocial disturbances with more severe irritable bowel syndrome (IBS) is well recognized. However, there is no evidence as to how these associations might be mediated. Functional magnetic resonance imaging (fMRI) offers an opportunity to study whether activation of the cingulate cortex, an area involved with the affective and pain intensity coding might be linked to poorer clinical status with IBS. In this case report, we found an association between the severity of a patient's clinical symptoms and psychosocial state, with activation of the cingulate cortex. We also found that clinical and psychosocial improvement was associated with reduced cingulate activation. Methods: Observational case report of a young woman observed for 16 years with a history of sexual abuse, psychosocial distress, and functional GI complaints. Psychosocial, clinical, and fMRI assessment was performed when the patient experienced severe symptoms and again 8 months later when clinically improved. Results: During severe illness, the patient had major psychosocial impairment, high life stress, a low visceral pain threshold, and activation of the midcingulate cortex (MCC), prefrontal area 6/44, and the somatosensory cortex, areas associated with pain intensity encoding. When clinically improved, there was resolution in activation of these 3 areas, and this was associated with psychosocial improvement and an increased threshold to rectal distention. Conclusions: Activation of the MCC and related areas involved with visceral pain encoding are associated with poor clinical status in patients with severe IBS and psychosocial distress and appear to be responsive to clinical improvement.

7 he clinical association of sexual or physical abuse 1 with the severity of irritable bowel syndrome (IBS) symptoms and the clinical outcome is well recognized. ${ }^{1-3}$
However, to date there is no evidence as to how these associations are mediated. Recent imaging methods for evaluating the brain's response to visceral distention offer a physiological window to understand how the central nervous system (CNS) responds to and modulates the clinical experience of pain. ${ }^{4}$ Also, there is new evidence that the brain's response to visceral signals in patients with IBS differs from that in controls. For example, using positron emission tomography $(\mathrm{PET})^{5}$ and functional MRI (fMRI) ${ }^{6}$ to assess CNS responses to rectal distention, activation of the MCC differs between patients with IBS and normal subjects. The MCC regulates skeletomotor activity and codes for the intensity of noxious stimulation. $^{7-9}$

We have recently shown that persons with a history of severe sexual and physical abuse with rectal distention activate the perigenual anterior cingulate cortex (pACC), which is involved in regulating autonomic activity and emotion, ${ }^{10}$ and this may amplify the enhanced cingulate response to visceral pain noted in patients with IBS. 5,6 Psychosocial factors may alter the experience of visceral pain by activating these specific areas of the brain. This case history reports associations between CNS cingulate activation from visceral distention, pain related to IBS, and psychometric evidence for severe psychosocial distress in a young woman suffering from IBS and sexual and emotional abuse. From our previous work, ${ }^{10}$ we

Abbreviations used in this paper: ACC, anterior cingulate cortex; CNS, central nervous system; fMRI, functional magnetic resonance imaging; IBS, irritable bowel syndrome; MCC, midcingulate cortex; pACC, perigenual anterior cingulate cortex; PET, positron emission tomography.

(C) $\mathbf{2 0 0 3}$ by the American Gastroenterological Association 0016-5085/03/\$30.00 doi:10.1053/gast.2003.50103 
hypothesized that this patient would show cingulate activation associated with high pain scores to rectal distention and abnormal psychosocial scores. Furthermore, when undertaking a second brain imaging study when her clinical and psychosocial status improved, we hypothesized that we would find an associated reduction in the degree of cingulate activation.

\section{Case Report}

\section{Childhood History}

The patient (A.L.) initially presented to the first author (D.A.D.) in May 1986, when at age 12 she was hospitalized on the pediatric unit of the University of North Carolina Hospital for severe constipation. She first developed symptoms at age 7 . They were characterized by lower abdominal pain and infrequent, painful passage of hard stools every 10-12 days, and occasionally, when symptoms were severe, episodes of nausea and vomiting. She also had asthma that was intermittently treated with bronchodilators. The medical evaluation was normal except for a large rectum and sigmoid colon noted on colonoscopy, and Hirschsprung's disease was ruled out by a normal anorectal manometry and giant forceps biopsy of the rectum that showed ganglion cells. She was discharged with a diagnosis of colonic inertia, and mineral oil was prescribed. Over the next several years, the first author saw her in clinic every 3-4 months. Her abdominal pain ameliorated, and she reported 2-3 nonpainful bowel movements every week. She did well in school, was socially active, and became a junior high school cheerleader.

On a visit in mid-1989, at age 16, A.L. acknowledged being sexually active with a 19-year-old male without using contraception. The first author suggested that she come back in a month to undergo a pelvic examination and to discuss birth control options. On the return visit, her condition had deteriorated. She was not eating well because of postprandial pain and nausea, and she had lost 4 pounds. She was feeling so poorly that she gave up cheerleading, and her grades were falling. She disclosed that after the previous clinic visit, she started having nightmares that gradually led to the recollection that from age $3-7$, she had been sexually abused by a family friend. Efforts to prosecute the perpetrator were unsuccessful. At the time, A.L.'s mother advised A.L. to "forget about" these traumatic events. Of note, it was at this time that A.L.'s symptoms of abdominal pain and constipation began. Over the next several years, A.L. was preoccupied with the bowel symptoms, and she had no recollection of the earlier traumatic events. Only many years later, when the first author suggested that she return to the clinic for a pelvic examination, was her recall of the prior traumatic memories triggered. Over the next several visits, the patient discussed these traumatic experiences, which seemed to help reduce her feelings of shame, vulnerability, and responsibility. Concurrently, a gradual improvement in her GI symptoms occurred, and she declined additional mental health counseling. By the winter of 1991, her symptoms were much improved, and the visits were discontinued.

\section{Adult History}

A.L. returned 9 years later at age 27 . Her medical health had been good except for occasional hospitalizations for asthma, and 1 episode in 1994 of possible autoimmune hepatitis with hepatomegaly. The abnormal liver chemistry studies cleared after a brief course of prednisone. The reason for the current visit related to a 6-month history of diarrhea-predominant IBS. Symptoms began promptly after the patient developed a Clostridium diffcile colitis from amoxicillin/clavulanate therapy prescribed for a severe asthma attack. Metronidazole and vancomycin eradicated the bowel infection, but the postprandial lower abdominal pain with diarrhea continued.

The patient's psychosocial history included the posttraumatic stress disorder resulting from her childhood abuse, and the development in late adolescence of obsessive-compulsive disorder, treated briefly with paroxetine. In addition, there was a more recent history of emotional and sexual abuse developing during her 3-year-long marriage. A.L. believed that her history of abuse interfered with her current interactions with her husband. She felt less able to assert her views or defend herself from his advances. Over the last several months, this situation had worsened, exacerbating her bowel symptoms. Fluoxetine, $20 \mathrm{mg}$, and alosetron, $1 \mathrm{mg}$, were prescribed, and the patient was referred to a psychologist for cognitivebehavioral treatment in addition to ongoing care from the first author.

Over the next year, little improvement occurred. A.L. surreptitiously obtained narcotics for pain control from other physicians, and the pain, nausea, and occasional vomiting and diarrhea continued to be of moderate severity. Her home situation remained difficult, and although she talked about leaving her husband, she was unable to do so. She reported feelings of depression, helplessness, vulnerability, poor self-esteem, and catastrophic thoughts, stating that she could not continue to endure the symptoms and emotional distress. In addition, she stopped seeing her therapist. 


\section{Severe IBS / Psychological Distress}

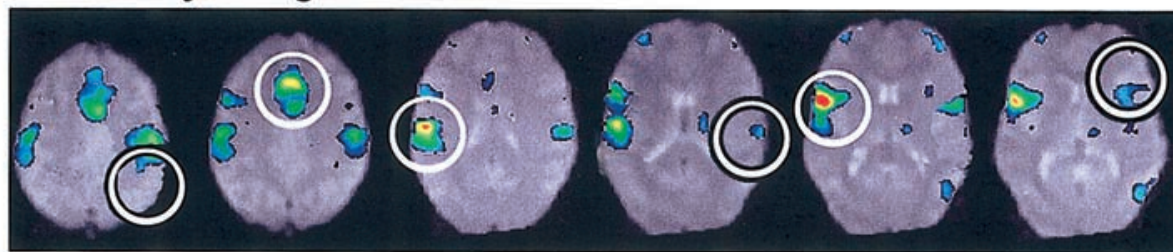

Clinical Recovery (8 months later)
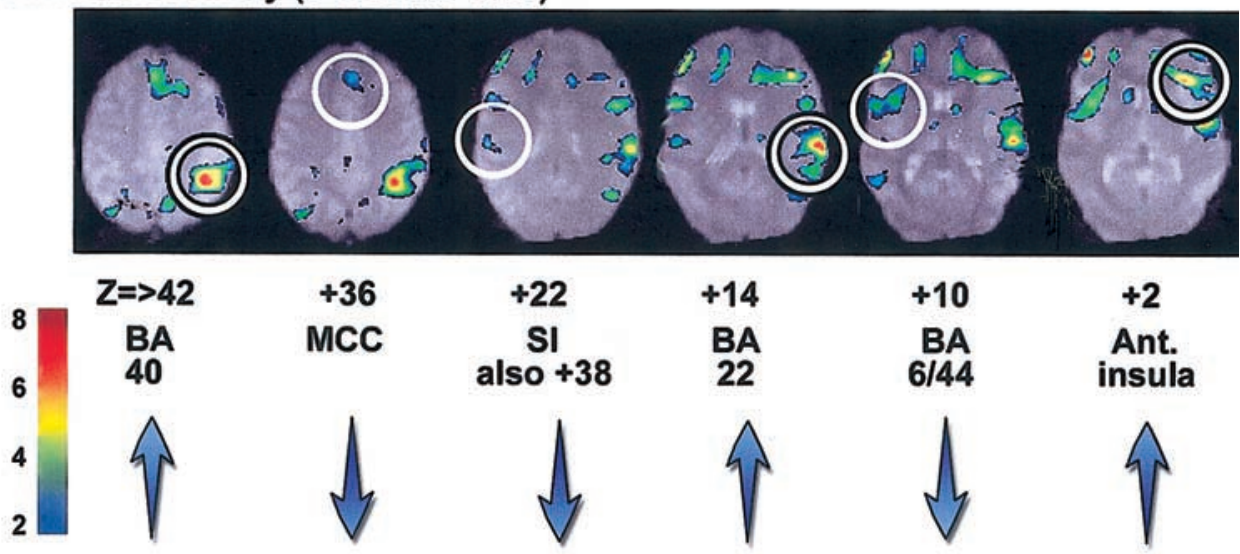

Figure 1. Pairs of horizontal sections from the severe IBS/psychological distress (top) and clinical recovery ( 8 months later) (bottom) follow-up fMRI studies. Images are a subtraction of a BOLD signal generated during baseline rectal distention $(15 \mathrm{~mm} \mathrm{Hg})$ from that at $50 \mathrm{~mm} \mathrm{Hg}$ distention. Each presymptomatic and postsymptomatic pair of sections was selected to show a single cortical area difference; that is, there were other significant ${ }^{33}$ changes in the same and other sections. The $Z$ scores were matched to the standardized atlas, ${ }^{12}$ and the significance of $Z$ scores is shown with standard color coding (red for highest to blue for lowest). Cortical areas with high initial activation during the "severe IBS" state on top are circled in white and include the MCC, primary somatosensory cortex, and prefrontal area 6/44. Areas with initially low levels of activation that were significantly elevated at clinical recovery (8 months later) are highlighted with black-stroked circles and included areas 40 and 22 and the anterior insula. Reductions in cortical activity that may account for resolution of stimulus-evoked pain and psychosocial symptoms are the MCC, primary somatosensory cortex, and prefrontal area 6/44.

\section{First Brain Imaging Study}

In August 2001, A.L. volunteered to participate in a research protocol that involved psychosocial assessment and fMRI brain imaging. All images were acquired on a Siemens 1.5 Tesla MR scanner (Siemens Medical Systems, Munich, Germany) with a gradient strength of 25 mini Tesla per meter $(\mathrm{mT} / \mathrm{m})$. A T2*-weighted echo planar imaging sequence was used to acquire images. The imaging parameters were as follows: repetition time, 3 seconds; echo time, $54 \mathrm{msec}$; slice thickness, $5 \mathrm{~mm}$; field of view, $192 \times 192 \mathrm{~mm}^{2}$, with a matrix size of $64 \times 64$; and 16 slices. Images were obtained after 4 sequences (ON/OFF) of $15 \mathrm{~mm} \mathrm{Hg}$ and then $50 \mathrm{~mm} \mathrm{Hg}$ rectal distentions. A block design was used with 39 seconds for the resting (OFF) and rectal balloon distention $(\mathrm{ON})$ states. Statistical parametric mapping (SPM) 99 software $^{11}$ (Wellcome Department of Imaging Neuroscience, University College, London, England) was used for motion correction, spatial filtering (full width at half-maximum, $10 \mathrm{~mm}$ ), alignment with the Talairach space, and statistical analysis.
A.L. first reported the $15-\mathrm{mm}$ distentions as moderately severe (3 on a scale of $1-5$ ), and with the first 50 $\mathrm{mm} \mathrm{Hg}$ distention, she stopped the study, reporting unbearably severe pain (intensity score of 5). However, she then agreed to continue the study; the fMRI images at $50-\mathrm{mm} \mathrm{Hg}$ distention are shown in the top row in Figure 1. The most prominent activity was in the MCC, prefrontal area $6 / 44$, and the somatosensory cortex. The Talairach and Tournoux ${ }^{12}$ coordinates and $\mathrm{Z}$ scores of these 3 areas of activation are given in Table 1 (visit 1 ).

\section{Clinical Recovery}

Several weeks after the brain imaging study, the family held a confrontation session to address A.L.'s narcotic use. She entered, and successfully recovered from, an inpatient detoxification program and returned to weekly counseling. Several weeks later, she separated from her husband and began divorce proceedings. Over the next several months, she reported increased selfconfidence and self-esteem, and less depression. Her daily function and quality of life improved, and she discontin- 
Table 1. Areas and Z Scores of Activations

\begin{tabular}{lrrrrrr}
\hline & \multicolumn{3}{c}{ Coordinates } & & \multicolumn{2}{c}{$Z$ scores } \\
\cline { 2 - 3 } \cline { 7 - 7 } \multicolumn{1}{c}{ Area } & $X$ & $Y$ & $Z$ & & Visit \#1 & Visit \#2 \\
\hline MCC & 0 & 26 & 36 & 6.6 & 0 \\
Somatosensory & & & & & \\
$\quad$ cortex & -58 & -18 & 22 & 6.2 & 0 \\
$6 / 44$ & -52 & 8 & 10 & 6.5 & 3.6 \\
40 & 44 & -46 & 42 & & 2.6 & 5.3 \\
22 & 60 & -26 & 14 & & 2.4 & 4.9 \\
ant. insula & 36 & 26 & 2 & 0 & 4.1 \\
\hline
\end{tabular}

NOTE. Based on a small-volume correction of a 15-mm diameter circle in SPM99; $P=0.01$ uncorrected.

ant. insula, anterior insula.

ued narcotics. Concurrently, her pain and diarrhea resolved almost completely.

\section{Second Brain Imaging Study}

In April 2002, 8 months after the first study and during a period of clinical improvement, A.L. underwent a second brain imaging study using the same distention protocol. She now reported the $15-\mathrm{mm}$ distentions as mild (intensity score of 1) and the 50-mm distentions as moderate (intensity score of 3). The fMRI images at the $50 \mathrm{~mm} \mathrm{Hg}$ rectal distention are shown in Figure 1 (bottom). At this time, there was minimal activation of the MCC and smaller reductions in activity in the primary somatosensory cortex and frontal area 6/44. In addition, there was marked increased activation of the right posterior parietal area 40 , not detected on the first scan, and increases in the superior temporal area 22 and right anterior insula. The Talairach coordinates and $\mathrm{Z}$ scores for these areas of activation are given in Table 1 (visit 2).

\section{Psychosocial and Behavioral Assessment}

Within a few days of each brain imaging session, A.L. underwent a psychological interview and filled out psychosocial questionnaires. The results, shown in Table 2, compare A.L.'s scores at study 1 (severe IBS symptoms) and study 2 (mild IBS symptoms) with the mean scores of 431 patients with moderate to severe IBS seen at the University of North Carolina and University of Toronto. At study 1, A.L.'s scores on the SCL-90 (a tool for assessing psychological distress ${ }^{13}$ ) were markedly elevated, at the 70th percentile for female psychiatric outpatients. Similarly, her depression score using the Beck II depression scale ${ }^{14}$ was at the most severe range. The severity of stressful life events assessed by the Stressful Life Events and Difficulties Interview ${ }^{15}$ was very high
(13). With regard to coping style, her catastrophizing score was higher and the perceived ability to control and decrease symptoms lower (i.e., poorer) than the scores of the comparison group. ${ }^{16}$ Health care utilization measures were also considerably worse than those of the comparison group. At the second study, at the time of clinical recovery, A.L. demonstrated marked improvement in all psychological scores, with values close to or within normal ranges. Notably, health care utilization dropped from 3 to 0 hospitalizations in the previous 3 months, and the number of days in bed was reduced by $33 \%$. The number of physician visits remained the same, but these visits were scheduled as part of the treatment program and were initiated by A.L. and the physician.

\section{Discussion}

This case report of a young woman with functional GI symptoms and abuse history uniquely demonstrates an association between psychosocial distress, GI symptoms, tolerance to rectal distention, and changes in brain activation as demonstrated by fMRI. There are 2 levels of association to consider: (1) clinical association between historical events and GI symptoms and (2) physiological associations between brain activation, rectal distention pain, and psychosocial scores.

\section{Clinical Association Between Historical Events and GI Symptoms}

The nature and severity of A.L.'s chronic symptoms were temporally related to environmental and psy-

Table 2. Psychosocial/Behavioral Scores at Study 1, Study 2, and With IBS Comparison Group

\begin{tabular}{|c|c|c|c|}
\hline Measure & $\begin{array}{c}\text { Study } 1 \\
\text { Severe IBS }\end{array}$ & $\begin{array}{c}\text { Study } 2 \\
\text { Minimal IBS }\end{array}$ & $\begin{array}{c}\text { IBS Means } \\
( \pm \mathrm{SD}) \\
\text { UNC-Toronto } \\
\text { Mod-severe IBS } \\
(\mathrm{n}=431)\end{array}$ \\
\hline SCL-90-Global score & 2.62 & 0.47 & $0.70( \pm 0.51)$ \\
\hline SCL-90-Depression & 3.62 & 0.38 & $0.96( \pm 0.72)$ \\
\hline SCL-90-Anxiety & 3.20 & 1.20 & $0.63( \pm 0.60)$ \\
\hline Beck II depression & 36 & 6 & $10.5( \pm 8.36)$ \\
\hline No. of stressors $/ 3 \mathrm{mo}$. & 13 & 7 & \\
\hline Catastrophizing score & 12 & 7.02 & $10.02( \pm 7.41)$ \\
\hline Ability to control sx. (0-6) & 2 & 4 & $2.51( \pm 1.24)$ \\
\hline $\begin{array}{l}\text { Ability to decrease sx. } \\
\qquad(0-6)\end{array}$ & 2 & 3 & $2.20( \pm 1.18)$ \\
\hline $\begin{array}{l}\text { Days in bed } / 3 \text { months } \\
\text { Hospitalizations } / 3\end{array}$ & 15 & 10 & $6.24( \pm 12.0)$ \\
\hline months & 3 & 0 & $0.04( \pm 0.30)$ \\
\hline MD visits $/ 3$ months & 4 & 4 & $3.61( \pm 5.14)$ \\
\hline
\end{tabular}

Sx, symptoms. 
chosocial events. A.L. had a psychological history most consistent with posttraumatic stress disorder, in which childhood abuse is experienced as a threat to one's integrity and is associated with feelings of fear, vulnerability, and helplessness. This can lead to repression of memories for many years, with later emergence often after a triggering event (in this case, the anticipation of a pelvic examination) taking the form of nightmares, flashbacks, autonomic arousal, and physical and psychological symptoms. ${ }^{17}$ Notably, A.L. developed abdominal pain and colonic inertia after the 4-year history of sexual abuse, precisely at the time she began to repress the traumatic memories. This is consistent with a study by Felice et al. ${ }^{18}$ that prospectively followed 25 preadolescent and adolescent rape victims over several years and reported "an initial development of fears and phobias, followed later by the denial of problems, and still later by the emergence of psychosomatic problems." Other studies indicate that GI symptoms are the most commonly reported complaints among female abuse victims, ${ }^{19}$ and among victims of severe abuse, IBS and other functional GI disorders are the GI diagnoses most frequently identified. ${ }^{3,19}$ After A.L.'s memories of the abuse returned, addressing her thoughts and feelings during the therapeutic interaction led to improvement in her emotional distress, and the symptoms ameliorated for almost 10 years.

Notably, A.L.'s GI symptoms recurred as IBS in adulthood after $C$. difficile infection from antibiotics administered to treat her asthma. This was at a time when she reexperienced sexual and emotional abuse from her marriage. Gwee et al. ${ }^{20}$ have reported that psychological distress at the time of an acute enteric infection predicts the later development of postinfectious IBS. It is hypothesized that psychological distress leads to the perpetuation of these symptoms, either through stress-mediated activation of peripheral neuroimmune-inflammatory pathways or through stress-mediated failure of the CNS to down-regulate incoming visceral afferent signals, thereby amplifying GI symptoms. ${ }^{21}$ In this regard, it is notable that A.L.'s symptoms again improved after she divorced and removed herself from the abusive situation, and started psychotherapy.

Finally, A.L.'s nausea, vomiting, and abdominal pain may have been more severe and more difficult to manage because of her concurrent and surreptitious use of narcotics. The narcotic bowel syndrome, in which frequent and prolonged use of narcotics leads to worsening abdominal pain, nausea, and vomiting, is an often over- looked condition. ${ }^{22}$ It is very possible that A.L.'s withdrawal from narcotics also contributed to the dramatic improvement in her symptoms. ${ }^{23}$

\section{Physiological Associations Between Brain Activation, Rectal Distention Pain, and Psychosocial Scores}

Despite common acceptance of the fact that psychosocial trauma and psychological distress can adversely affect clinical outcomes in IBS and other functional GI disorders, ${ }^{2,3,24}$ the physiological mechanisms behind this observation have not been established. Stress may amplify GI symptoms through various mechanisms, including increased autonomic reactivity, CNS-related (e.g., via CRF enhancement of motility or mucosal sensitivity via inflammatory/immune mechanisms), or amplification of central responses to afferent visceral signals via alterations in CNS pain regulatory pathways. ${ }^{25}$

This case report also demonstrates a possible link between the patient's changing psychosocial and emotional state, her level of pain sensitivity, and the nature and degree of physiological activation of her brain to visceral distention. Recent studies using PET $^{5}$ and $\mathrm{fMRI}^{6}$ have shown that patients with IBS have greater activation of the MCC relative to controls in response to rectal distention, although there has been a report of reduced activity in the ACC. ${ }^{26}$

The MCC has a well-documented role in skeletomotor regulation and intensity coding of noxious stimuli. ${ }^{7-9}$ Furthermore, the findings from our research group also suggest that in response to rectal distention, persons with histories of severe sexual and physical abuse preferentially activate the perigenual part of the $\mathrm{ACC}^{10,27}$ (a limbic structure that has a well-documented role in autonomic regulation and emotion), and activity in this region is enhanced during acute noxious cutaneous stimulation $^{28}$ and in chronic pain states. ${ }^{29,30}$ Thus it is possible that the enhanced MCC and pACC response to visceral pain noted in patients with IBS and abuse may be amplified by the effects of psychosocial trauma in at least a subgroup of patients with IBS. Further studies are needed with a larger sample of patients to sort out the specific roles of these 2 subregions of the cingulate cortex in IBS and abuse. Unfortunately, previous brain imaging studies of patients with IBS have not been evaluated for a history of sexual or physical abuse. However, this region is similar to a region that has reduced blood flow during symptom provocation in patients with posttraumatic stress disorder. ${ }^{31}$

Given this existing knowledge, we assumed that in this patient with a history of abuse, high emotional 
distress would be associated with greater symptom severity. Furthermore, we hypothesized that these clinical features would correlate during rectal distention with changes in brain activity in those regions related to visceral pain. We also hypothesized that with a reduction in emotional distress, these findings would be reversed. During A.L.'s first fMRI session, when her physical and psychological symptoms were severe and associated with maladaptive coping and marked illness-related behaviors, the most prominent activity (Figure 1, top) was noted in the MCC, and prefrontal area 6/44 and the primary somatosensory cortex.

The second imaging session occurred after the abusive relationship had been terminated and the IBS symptoms had resolved concurrent with near normalization of her psychological scores. At this time, there was a marked reduction in activation of the MCC concurrent with reduced activity in area 6/44 and the primary somatosensory cortex. Because the same level of bowel distention $(50 \mathrm{~mm} \mathrm{Hg})$ was associated with at first severe, then milder levels of pain during this scanning, it is likely that this reduction, particularly in MCC activity, contributes significantly to the altered perception of pain. The reduced activation of area 6/44 in the second session was not surprising, because this area has the highest correlation with pain intensity ratings of all cortical areas studied. ${ }^{9}$

The increases in cortical activity in other areas of the brain during the second session are also associated with A.L.'s improvement (Figure 1, bottom), although the significance of these effects is uncertain and requires further confirmation. The marked activation of the right posterior parietal area 40 present in the second session was not detected during the first session. There was also a major increase in superior temporal area 22 and in the anterior insula. Activity in posterior parietal cortex has been associated with the perception of self ${ }^{32}$ and that the anterior insula is involved in an overall sense of the body. ${ }^{33}$ To the extent that MCC activation mediates enhanced or abnormal sensory and motivational perceptions in the anticipation of noxious/uncomfortable bowel activity, ${ }^{5}$ it is also likely that during the period of clinical recovery, the enhancement of areas 40 and 22 and the anterior insula activity may be involved in selforientation to somatic and autonomic functions, and also to the interpretation of such signals as not threatening. Thus these latter physiological responses could be an adaptive means of reorienting cortical function away from misinterpreting internal states, including bowel events, from the cingulate cortex, and this reorientation could be part of the therapeutic response. Because this is the first report of elevated cortical responses as part of a therapeutic response, these concepts are hypothetical and require confirmation with future studies.

Certain qualifications with regard to our interpretation of the data need to be considered. First, these physiological observations possibly may be influenced by the patient's use of narcotics during the first brain imaging session, taken without our knowledge. However, binding of opiates such as diprenorphine is highest in pACC, ${ }^{34}$ and the major area of activation in our case was in the MCC. In addition, the primary somatosensory cortex and area 6/44 have low opiate binding, thus making it less likely that A.L.'s narcotic use explained the areas of activation noted in this case report. Second, these findings possibly may relate to a change in nociception in the viscera independent of, or in addition to, central influences. Although there can be a waxing and waning of symptoms in IBS, there is little evidence to suggest that this occurs by peripheral rather than central mechanisms. With regard to peripheral effects in this patient, if they were related to narcotic use, then there would likely have been a reduction in peripheral pain signals (in addition to central processing); however, the patient took narcotics during the first study, when the pain was far greater. Furthermore, sensitization from postinfectious IBS from $C$. difficile could resolve over time, yet this patient's infection and subsequent IBS occurred 2 years before the first brain imaging study, when the clinical findings and high pain reports were still present, thus making it unlikely that the sensitization would have resolved spontaneously between 2 years and 2 years and 8 months later. In comparison, by the second brain imaging study, the patient had experienced major changes in emotional and cognitive status that have been found to affect clinical pain reports, including reduced psychological distress (e.g., anxiety and depression), life stress, catastrophizing scores, and improved cognitions relating to the ability to control and decrease symptoms. ${ }^{2}$ These findings, coupled with the fact that anxiety and cognitive factors (e.g., hypnosis and distraction) can reduce pain reports and affect brain activation, compel us to exclude the possibility of a peripheral mechanism to explain her improvement, or at least to not accept that peripheral responsiveness plays a major role in our findings. Finally, it is possible that the repeated rectal distention protocol during the second session confounded the findings due to patient anticipation. However, it has been shown that anticipation of visceral stimulation in IBS enhances MCC activity; 5 thus, antic- 
ipatory effects are unlikely to account for observations in this case.

In conclusion, we have documented in a woman with a history of sexual abuse and fluctuating severe functional bowel symptoms, an association between psychosocial distress, increased bowel symptoms, lower sensation threshold to rectal distention, and increased activation of the MCC, an area associated with intensity coding of noxious stimulation. Furthermore, we have found resolution of these brain activation patterns associated with improvement in psychological state and physical symptoms. This finding suggests that brain activation to rectal distention may vary with emotional status, rather than being a feature of the individual or disease (e.g., IBS). Because this is the first clinical report involving only 1 patient to examine the responsiveness of CNS activation to changes in severity of the patient's emotional and physical state, the findings are only preliminary. Furthermore, as a case report, the findings do not exclude other explanations in addition to, or at the exclusion of, changes in psychosocial state, such as adaptation or habituation to the fMRI environment, peripheral changes in the gut affecting sensitivity, or other undetermined changes over time. Therefore, future controlled studies involving a large number of patients are needed to sort out the validity, specificity, and generality of these findings. Such studies may help determine whether CNS activation could be used as a marker for clinical response to therapeutic interventions, including antidepressants and cognitive behavioral treatments in the care of patients with severe functional GI disorders.

\section{References}

1. Drossman DA, Creed FH, Olden KW, Svedlund J, Toner BB, Whitehead WE. Psychosocial aspects of the functional gastrointestinal disorders. Gut 1999;45:|I25-II30.

2. Drossman DA, Creed FH, Olden KW, Svedlund J, Toner BB, Whitehead WE. Psychosocial aspects of the functional gastrointestinal disorders. In: Drossman DA, Corazziari E, Talley NJ, Thompson WG, Whitehead WE, eds. Rome II. The functional gastrointestinal disorders: diagnosis, pathophysiology and treatment, a multinational consensus. 2nd ed. McLean, VA: Degnon and Associates, 2000:157-245.

3. Drossman DA, Li Z, Leserman J, Toomey TC, Hu Y. Health status by gastrointestinal diagnosis and abuse history. Gastroenterology 1996;110:999-1007.

4. Ringel $Y$, Drossman DA. From gut to brain and back-a new perspective into functional gastrointestinal disorders. J Psychosom Res 1999;47:205-210.

5. Naliboff BD, Derbyshire SWG, Munakata J, Berman S, Mandelkern M, Chang L, Mayer EA. Cerebral activation in irritable bowel syndrome patients and control subjects during rectosigmoid stimulation. Psychosom Med 2001;63:365-375.

6. Mertz H, Morgan V, Tanner G, Pickens D, Price R, Shyr Y, Kessler R. Regional cerebral activation in irritable bowel syndrome and control subjects with painful and nonpainful rectal distention. Gastroenterology 2000;118:842-848.

7. Vogt BA, Vogt LJ, Nimchinsky EA, Hof PR. Primate cingulate cortex chemoarchitecture and its disruption in Alzheimer's disease. In: Bloom FE, Bjorkland A, Hokfelt T, eds. Handbook of chemical neuroanatomy, vol. 13: the primate nervous system, part I. Amsterdam: Elsevier, 1997:455-528.

8. Derbyshire SWG, Jones AKP, Gyulai F, Clark S, Townsend D, Firestone LL. Pain processing during three levels of noxious stimulation produces differential patterns of central activity. Pain 1997;73:431-435.

9. Coghill RC, Sang CN, Maisog JMA, ladarola MJ. Pain intensity processing within the human brain: a bilateral, distributed mechanism. J Neurophysiol 1999;82:1934-1943.

10. Ringel Y, Drossman DA, Liu H, Lin W, Smith JK, Keeter L, An H, Vogt B, Leserman J, Whitehead WE. fMRI of cingulated activation to painful rectal distention in IBS and sexual/physical abuse (abstr). Gastroenterology 2002;122:A311.

11. Friston KJ, Ashburner J, Frith CD, Poline JB, Heather JD, Frackowiak RSJ. Spatial registration and normalization of images. Hum Brain Mapp 1995;2:165-189.

12. Talairach J, Tournoux P. Co-planar stereotaxic atlas of the human brain. New York: Stuttgart, 1988.

13. Derogatis LR. SCL-90-R: administration, scoring, and procedures manual II for the r(evised) version. Towson, MD: Clinical Psychometric Research, 1983.

14. Beck AT, Ward CH, Mendelson M, Mock J, Erbaugh J. An inventory for measuring depression. Arch Gen Psychiatry 1961;4:561571.

15. Leserman J, Petitto JM, Perkins DO, Folds JD, Golden RN, Evans DL. Severe stress, depressive symptoms, and changes in lymphocyte subsets in human immunodeficiency virus-infected men: a 2-year follow-up study. Arch Gen Psychiatry 1997;54:279-285.

16. Robinson ME, Riley JL III, Myers CD, Sadler IJ, Kvaal SA, Geisser ME, Keefe FJ. The Coping Strategies Questionnaire: a large sample, item-level factor. Clin J Pain 1997;13:43-49.

17. Lamprecht F, Sack M. Posttraumatic stress disorder revisited. Psychosom Med 2002;64:222-237.

18. Felice M, Grant J, Reynolds B, Gold S, Wyatt M, Heald FP. Follow-up observations of adolescent rape victims. Clin Pediatr 1978;17:311-315.

19. Drossman DA, Talley NJ, Olden KW, Leserman J, Barreiro MA. Sexual and physical abuse and gastrointestinal illness: review and recommendations. Ann Intern Med 1995;123:782-794.

20. Gwee KA, Leong YL, Graham C, McKendrick MW, Collins SM, Walters SJ, Underwood JE, Read NW. The role of psychological and biological factors in postinfective gut dysfunction. Gut 1999; 44:400-406.

21. Drossman DA. Mind over matter in the postinfective irritable bowel. Gut 1999;44:306-307.

22. Rogers M, Cerda JJ. The narcotic bowel syndrome (editorial). J Clin Gastroenterol 1989;11:132-135.

23. Sandgren JE, McPhee MS, Greenberger NJ. Narcotic bowel syndrome treated with clonidine. Ann Intern Med 1984;101:331334.

24. Drossman DA, Camilleri M, Mayer EA, Whitehead WE. AGA technical review on irritable bowel syndrome. Gastroenterology 2002; 123:2108-2131.

25. Drossman DA. A biopsychosocial understanding of gastrointestinal illness and disease. In: Feldman M, Scharschmidt B, Sleisenger $\mathrm{MH}$, eds. Sleisenger and Fortran's gastrointestinal disease. 7th ed. Philadelphia: Saunders, 2002:2371-2380.

26. Silverman DHS, Munakata JA, Ennes H, Mandelkern MA, Hoh CK, Mayer EA. Regional cerebral activity in normal and pathologic perception of visceral pain. Gastroenterology 1997;112:64-72.

27. Ringel Y, Drossman DA, Liu H, Lin W, Smith JK, Keeter L, An H, 
Vogt B, Leserman J, Whitehead WE. Anterior shift of cingulate activity with painful rectal distention in subjects with sexual/ physical abuse (abstr). Gastroenterology 2002;122:A311.

28. Vogt BA, Derbyshire S, Jones AKP. Pain processing in four regions of human cingulate cortex localized with co-registered PET and MR imaging. Eur J Neurosci 1996;8:1461-1473.

29. Derbyshire SWG. Exploring the pain "neuromatrix." Curr Rev Pain 2000;4:467-484.

30. Vogt BA, Sikes RW. The medial pain system, cingulate cortex, and parallel processing of nociceptive information. In: Mayer EA. Saper CB, eds. The biological basis for mind-body interactions. Los Angeles: Elsevier, 2000:223-235.

31. Shin LM, McNally RJ, Kosslyn SM, Thompson WL, Rauch SL, Alpert NM, Metzger LJ, Lasko NB, Orr SP, Pitman RK. Regional cerebral blood flow during script-driven imagery in childhood sexual abuse-related PTSD: a PET investigation. Am J Psychiatry 1999;156:575-584.

32. Vogt BA, Devinsky O. Topography and relationships of mind and brain. Prog Brain Res 2000;122:11-22.
33. Craig AD. An ascending general homeostatic afferent pathway originating in lamina I. Prog Brain Res 1996;107:225-242.

34. Vogt BA, Watanabe H, Grootoonk S, Jones AKP. Topography of iprenorphine binding in human cingulate gyrus and adjacent ortex derived from coregistered PET and MR images. Hum Brain Mapp 1995;3:1-12.

Received June 14, 2002. Accepted November 21, 2002.

Address requests for reprints to: Douglas A. Drossman, M.D., Division of Digestive Diseases, 726 Burnett-Womack Building, CB \#7080, University of North Carolina, Chapel Hill, North Carolina 27599-7080. e-mail: Drossman@med.unc.edu; fax: (919) 966-8929.

Supported in part by an educational grant from Solvay Pharmaceuticals.

Dr. Whitehead is a consultant on the Speaker's Bureau and a grant recipient for Norvartis, and is a consultant and grant recipient for GlaxoSmithKline.

The authors thank Robert S. Sandler for his helpful comments in the preparation of this manuscript. 